\title{
AN OSIRIS STUDY OF THE GAS KINEMATICS IN A SAMPLE OF UV-SELECTED GALAXIES: EVIDENCE OF "HOT AND BOTHERED" STARBURSTS IN THE LOCAL UNIVERSE
}

\author{
Antara R. Basu-Zych ${ }^{1}$, Thiago S. Gonçalves ${ }^{2}$, Roderik Overzier ${ }^{3}$, David R. LaW ${ }^{5,6}$, David Schiminovich ${ }^{1}$, \\ Tim Heckman ${ }^{4}$, Chris Martin $^{2}$, Ted WYder $^{2}$, and Matt O’Dowd $^{1}$ \\ ${ }^{1}$ Department of Astronomy, Columbia University, 550 West 120th Street, New York, NY 10027, USA; antara@ astro.columbia.edu,ds@ astro.columbia.edu, \\ matto@astro.columbia.edu \\ ${ }^{2}$ California Institute of Technology, MC 405-47, 1200 East California Boulevard, Pasadena, CA 91125, USA; tsg@ astro.caltech.edu, cmartin@ srl.caltech.edu, \\ wyder@ srl.caltech.edu \\ ${ }^{3}$ Max-Planck-Institut für Astrophysik, D-85748 Garching, Germany; overzier@MPA-Garching.MPG.DE \\ ${ }^{4}$ Center for Astrophysical Sciences, The Johns Hopkins University, 3400 N. Charles St., Baltimore, MD 21218, USA; heckman@pha.jhu.edu \\ ${ }^{5}$ Department of Physics and Astronomy, University of California, Los Angeles, CA 90095, USA; drlaw @ astro.ucla.edu \\ Received 2008 December 19; accepted 2009 May 29; published 2009 June 22
}

\begin{abstract}
We present data from Integral Field Spectroscopy for three supercompact UV-Luminous Galaxies (ScUVLGs). As nearby $(z \sim 0.2)$ compact $\left(R_{50} \sim 1-2 \mathrm{kpc}\right)$ bright Paschen- $\alpha$ sources, with unusually high star formation rates (SFR $=3-100 M_{\odot} \mathrm{yr}^{-1}$ ), ScUVLGs are an ideal population for studying detailed kinematics and dynamics in actively star-forming galaxies. In addition, ScUVLGs appear to be excellent analogs to high-redshift Lyman Break Galaxies (LBGs), and our results may offer additional insight into the dynamics of LBGs. Previous work by our team has shown that the morphologies of these galaxies exhibit tidal features and companions, and in this study we find that the dynamics of ScUVLGs are dominated by disturbed kinematics of the emission line gas-suggesting that these galaxies have undergone recent feedback, interactions, or mergers. While two of the three galaxies do display rotation, $v / \sigma<1$ - suggesting dispersion-dominated kinematics rather than smooth rotation. We also simulate how these observations would appear at $z \sim 2$. Lower resolution and loss of low surface brightness features cause some apparent discrepancies between the low- $z$ (observed) and high- $z$ (simulated) interpretations and quantitatively gives different values for $v / \sigma$, yet simulations of these low $z$ analogs manage to detect the brightest regions well and resemble actual high- $z$ observations of LBGs.
\end{abstract}

Key words: galaxies: interactions - galaxies: kinematics and dynamics - galaxies: starburst - ultraviolet: galaxies

\section{INTRODUCTION}

Across cosmic time, processes driving galaxy formation are expected to transition from hierarchical merging at early times to secular evolution at low redshift (White \& Rees 1978; Abadi et al. 2003; Kormendy \& Fisher 2005). In the case of hierarchical merging, galaxies grow as halos merge, causing disturbed kinematics and dynamics. Secular evolution describes slow and steady mechanisms, including gas accretion and internal processes, and is consistent with smooth rotation.

The Galaxy Evolution Explorer (GALEX) has uncovered a population of local $(z=0.1-0.3)$, intensely star-forming galaxies. These ultraviolet luminous galaxies (UVLGs) are selected based on their Far-UV (FUV) luminosities $\left(L_{\mathrm{FUV}}>\right.$ $\left.2 \times 10^{10} L_{\odot}\right)$. UVLGs exhibiting the highest surface brightnesses $\left(I_{\mathrm{FUV}}>10^{9} L_{\odot} \mathrm{kpc}^{-2}\right.$ ), named supercompact UVLGs (ScUVLGs), are ideal candidates for Integral-field Spectroscopy (IFS). They are bright sources whose compact sizes (with halflight radii $\approx 1-2 \mathrm{kpc}$ ) accommodate the limited fields of view (FOV) of many IFS instruments, typical redshifts place the Paschen- $\alpha(\operatorname{Pa} \alpha)$ line conveniently in the $K$-band filter, and unusually high star formation rates (SFRs), with the SFR = 3-100 $M_{\odot} \mathrm{yr}^{-1}$, designate them as a unique sample. These ScUVLGs bear striking resemblances to Lyman Break Galaxies (LBGs) - sharing similar specific SFRs, metallicities, kinematics, and attenuations (Heckman et al. 2005; Hoopes et al. 2007; Basu-Zych et al. 2007). When resimulated at high redshift, their morphologies are similar to those of LBGs (Overzier et al. 2008). ScUVLGs constitute a local $(z \sim 0.2)$ sample well

\footnotetext{
${ }^{6}$ Hubble Fellow.
}

suited for studying details of galaxy formation with very high physical resolution and sensitivity using IFS.

LBGs, selected by the Lyman break dropout technique (Steidel et al. 2000), form stars at intense rates, dominating the UV luminosity density at $z>2$. Bouwens et al. (2006) find only a modest decrease in the UV luminosity density out to $z=6$, indicating that LBGs represent a major phase in early stages of galaxy formation and evolution. Many studies have used IFS to observe the dynamics of LBGs, at $z=2-3$, aiming to pinpoint the dominant formation process for these galaxies (i.e., Law et al. 2007; D. R. Law et al. 2009, in preparation; Nesvadba et al. 2006; Förster Schreiber et al. 2006; Wright et al. 2007; Nesvadba et al. 2008; Stark et al. 2008). These studies probe key questions: what causes such intense star formation in these systems - disk instabilities or tidal shocks from mergers? How does angular momentum evolve in early star-forming galaxies? Yet, at the high redshifts of LBGs, spatial resolution and depth are fundamentally limited. Except in the fortuitous but rare cases of gravitationally lensed LBGs, IFS at $z>2$ may be biased toward brighter and larger LBGs whose dynamics differ from typical LBGs (Nesvadba et al. 2008; Stark et al. 2008).

We have observed three ScUVLGs with the OH Suppressing InfraRed Imaging Spectrograph (OSIRIS) instrument. ${ }^{7}$ While a larger sample of ScUVLGs has been commissioned for IFS

\footnotetext{
7 The data presented herein were obtained at the W. M. Keck Observatory, which is operated as a scientific partnership among the California Institute of Technology, the University of California and the National Aeronautics and Space Administration. The Observatory was made possible by the generous financial support of the W. M. Keck Foundation.
} 


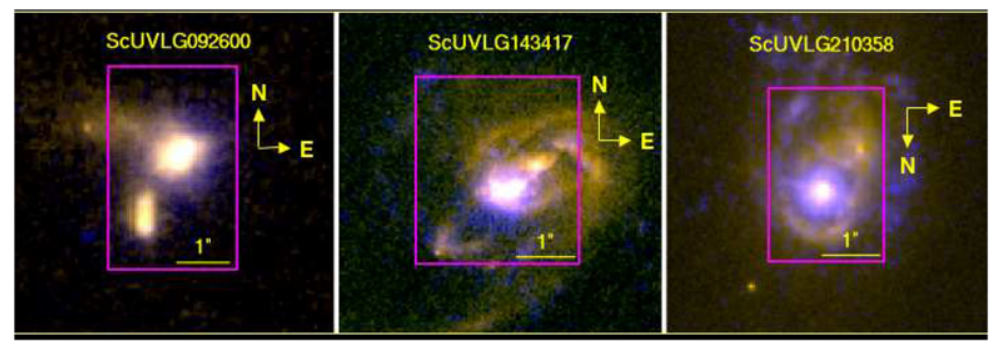

Figure 1. HST optical (red and green) + UV (blue) images (left to right; see Overzier et al. (2008) and R. A. Overzier et al. (2009, in preparation)): ScUVLG092600, ScUVLG143417, and ScUVLG210358. Magenta boxes mark OSIRIS FOV.

studies, in this Letter we share initial results and compare them with high- $z$ LBG studies. In Section 2, we discuss data reduction and analyze the results from these observations in Section 3. We discuss the relevance of this work, related to other high- $z$ LBG studies in the final section, Section 4. Throughout our analysis, we apply $\Lambda$ cold dark matter $(\Lambda C D M)$ cosmology with $\left(\mathrm{H}_{0}, \Omega_{M}\right.$, $\left.\Omega_{\Lambda}\right)=\left(70 \mathrm{~km} \mathrm{~s}^{-1} \mathrm{Mpc}^{-1}, 0.3,0.7\right)$.

\section{DATA AND ANALYSIS}

Using the $\mathrm{OH}$ Suppressing Infrared Imaging Spectroscope (OSIRIS; Larkin et al. 2006) instrument at the Keck II telescope, we observed SDSS J092600.40 + 442736.1 and SDSS $\mathrm{J} 143417.15+020742.5$ (referred to as ScUVLG092600 and ScUVLG143417, respectively) in Spring 2008 and SDSS J210358.74 - 072802.4 (ScUVLG210358, henceforth) in Fall 2008. The OSIRIS utilizes the Laser Guide Star Adaptive Optics (LGSAO) system (Wizinowich et al. 2006; van Dam et al. 2006) to improve spatial resolution.

Several criteria were applied in selecting the targets and observing configuration. Using the LGSAO requires that targets be within $60^{\prime \prime}$ of a bright $\left(M_{R} \lesssim 17 \mathrm{mag}\right)$ tip-tilt reference star. Certain filter and spatial pixel (spaxal) scale combinations result in excess thermal background and/or detector noise (Law et al. 2006). To optimize the sensitivity of our observations, we chose the Kn3 filter with 50 mas lenslet $^{-1}$ scale. For this combination, the resulting FOV for our observations was approximately $2^{\prime \prime} \times$ $3^{\prime \prime}$ (the actual FOV varied because of slight recentering during observations - see magenta boxes in Figure 1). The resolution and summary of our observations for each target are given in Table 1 . Our velocity resolution is approximately $34-35 \mathrm{~km} \mathrm{~s}^{-1}$. Total on-target integration times were $\approx 2 \mathrm{hr}$ per target.

As previously mentioned, ScUVLG selection depends on FUV luminosity and surface brightness exceeding $10^{10.3} L_{\odot}$ and $10^{9} L_{\odot} \mathrm{kpc}^{-2}$, respectively. Active galactic nuclei (AGNs) are excluded from this sample using two separate methods: radio luminosities (see Basu-Zych et al. 2007; Best et al. 2005), and optical emission lines (see Hoopes et al. 2007; Baldwin et al. 1981). The ScUVLG sample consists of compact, starburstdominated galaxies exhibiting signs of small-scale mergers or interactions only detected in the rest-frame optical at the resolution and sensitivity of the Hubble Space Telescope (HST; Overzier et al. 2008). Our three galaxies span stellar mass and SFR ranges of the full set of ScUVLGs. In Table 1, we list some of their physical properties. Stellar masses are determined by fitting the spectral energy distribution (SED) using unresolved Sloan Digital Sky Survey (SDSS) photometry (see Salim et al. 2005; Kauffmann et al. 2003), assuming a Kroupa (2001) initial mass function (IMF). The SFRs are unattenuated SFRs, determined from radio luminosities (see Basu-Zych et al. 2007). We estimate that typical uncertainties associated with the stellar mass and SFRs are $\sim 30 \%$ based on comparing different mass modeling methods and SFR indicators. Average values and standard deviations for these properties in the full sample of 45 ScUVLGs (studied in Basu-Zych et al. (2007)) are as follows: $\langle\mathrm{SFR}\rangle=21 M_{\odot} \mathrm{yr}^{-1}$ and $\sigma_{\mathrm{SFR}}=16 M_{\odot} \mathrm{yr}^{-1},\left\langle\log M_{*} / M_{\odot}\right\rangle=$ 10.0 and $\sigma_{\log M_{*}} / M_{\odot}=0.5$. While both ScUVLG143417 and ScUVLG210358 are dominated by luminous, compact UV and line-emitting regions, they contain a significant low surface brightness disk structure and are more massive than the typical ScUVLG.

We create three-dimensional data cubes of spatial versus spectral dimensions by using the Keck/OSIRIS data-reduction pipeline. The pipeline performs the following tasks: corrects detector nonlinearity to form a data cube with calibrated wavelength units, subtracts a sky frame from the science frame to combine separate exposures into a single mosaicked data cube, removes cosmic rays, and corrects for detector cross talk. A custom IDL routine determines a mask for good data based on a signal-to-noise $(\mathrm{S} / \mathrm{N})$ threshold set at $3 \sigma$. After boxcar smoothing by a kernel of three lenslets in the spatial dimensions to increase the $\mathrm{S} / \mathrm{N}$, Gaussians were fit to the spectral data to determine the peak, peak location, and FWHM of the $\mathrm{Pa} \alpha$ feature — creating S/N maps, velocity maps and velocity dispersion $(\sigma)$ maps, respectively. Resulting images for the three targets are shown in Figure 2 (with $\mathrm{Pa} \alpha \mathrm{S} / \mathrm{N}$ contours marked for reference). Figure 1 displays the orientation and FOV of these targets.

For ScUVLG092600, we also made position-velocity (PV) diagrams (shown in Figure 3) using the kpvslice tool in KARMA (Gooch 1996). An integrated spectrum combining all the unmasked data was created using custom IDL software. To calculate inclination, $i$, we assume that face-on $(i=0)$ disks have circular isophotes and edge-on disks have intrinsic axis ratios of $0.2{ }^{8}$ Using $\mathrm{Pa} \alpha \mathrm{S} / \mathrm{N}$ maps, we use ratios of minor-to-major axes of the isophotes to measure inclinations. We use several isophote levels to determine the uncertainty of this measurement.

\section{RESULTS}

HST images (shown in Figure 1; see Overzier et al. (2008) and R. A. Overzier et al. (2009, in preparation)) reveal companions and disturbed morphologies, such as tidal features and plumes. Using IFS, we analyze the velocity structure for signs of smooth rotation. In two cases, we see evidence of regular rotation (ScUVLG092600 and ScUVLG210358), while ScUVLG143417 has no clearly defined velocity structure. However, even in the cases of ordered velocity, we find high dispersion $(v / \sigma \lesssim 1)$,

\footnotetext{
8 While typical spiral galaxies have intrinsic axis ratios $\left(q_{0}\right)$ of 0.2 , ScUVLGs may be thicker. We note that even for $q_{0}=0.5$, values of $v_{c}$ and $v / \sigma$ differ by $\lesssim 10 \%$, within measurement uncertainties.
} 
Table 1

Summary of Observations

\begin{tabular}{|c|c|c|c|c|c|c|c|c|c|c|c|c|c|c|c|c|}
\hline \multirow[t]{2}{*}{ Target } & \multirow[t]{2}{*}{ R.A. } & \multirow[t]{2}{*}{ Decl. } & \multirow[t]{2}{*}{$\mathrm{z}$} & \multirow{2}{*}{$\begin{array}{c}\theta_{\mathrm{PSF}}{ }^{\mathrm{a}} \\
\left({ }^{\prime \prime}\right)\end{array}$} & \multirow{2}{*}{$\begin{array}{c}\mathrm{SFR}^{\mathrm{b}} \\
\left(M_{\odot} \mathrm{yr}^{-1}\right)\end{array}$} & \multirow{2}{*}{$\begin{array}{c}\log \left(M_{*}\right) \\
\left(M_{\odot}\right)\end{array}$} & \multicolumn{5}{|c|}{ Observed Data } & \multicolumn{5}{|c|}{ High- $z$ Simulations } \\
\hline & & & & & & & Axis Ratio & $\begin{array}{c}i \\
\left({ }^{\circ}\right) \\
\end{array}$ & $\begin{array}{c}v_{c} \\
\left(\mathrm{~km} \mathrm{~s}^{-1}\right) \\
\end{array}$ & $\begin{array}{c}\sigma \\
\left(\mathrm{km} \mathrm{s}^{-1}\right) \\
\end{array}$ & $v / \sigma$ & Axis Ratio & $\begin{array}{c}\mathrm{i} \\
\left({ }^{\circ}\right) \\
\end{array}$ & $\begin{array}{c}v_{c} \\
\left(\mathrm{~km} \mathrm{~s}^{-1}\right) \\
\end{array}$ & $\begin{array}{c}\sigma \\
\left(\mathrm{km} \mathrm{s}^{-1}\right) \\
\end{array}$ & $v / \sigma$ \\
\hline ScUVLG092600 & 141.502 & 44.460 & 0.181 & $0.101(0.208)$ & 8 & $9.20^{\mathrm{c}}$ & $0.78 \pm 0.05$ & $40 \pm 5$ & $31_{-3}^{+4}$ & $84 \pm 3$ & $0.37 \pm 0.05$ & $0.90 \pm 0.05$ & $26 \pm 4$ & $59_{-7}^{+11}$ & $59 \pm 17$ & $1.0_{-0.6}^{+0.3}$ \\
\hline ScUVLG143417 & 218.571 & 2.128 & 0.180 & $0.098(0.197)$ & 15 & $10.89^{\mathrm{c}}$ & . & & 60 & $86 \pm 3$ & $<0.70$ & $\ldots$ & & 35 & $98 \pm 12$ & $<0.35$ \\
\hline ScUVLG210358 & 315.995 & -7.467 & 0.137 & $0.065(0.144)$ & 68 & $11.05^{\mathrm{d}}$ & $0.65 \pm 0.05$ & $51 \pm 4$ & $186_{-9}^{+12}$ & $175 \pm 4$ & $1.1 \pm 0.1$ & $0.79 \pm 0.02$ & $39 \pm 2$ & $167_{-7}^{+8}$ & $175 \pm 11$ & $0.9 \pm 0.1$ \\
\hline
\end{tabular}

Notes.

${ }^{a} \theta_{\text {PSF }}$ gives observed FWHM of the PSF, spatially smoothed FWHM is given in the parentheses.

b The SFR was determined from 1.4 GHz-derived SFR - proxy for total, unattenuated SFR (see Basu-Zych et al. (2007)).

${ }^{c} M_{*}$ based on SED-fitting (see Salim et al. 2005).

d $M_{*}$ is dust-corrected median stellar mass (Kauffmann et al. 2003) from MPA/JHU value added catalog (see http://www.mpa-garching.mpg.de/SDSS/index_old.html). 


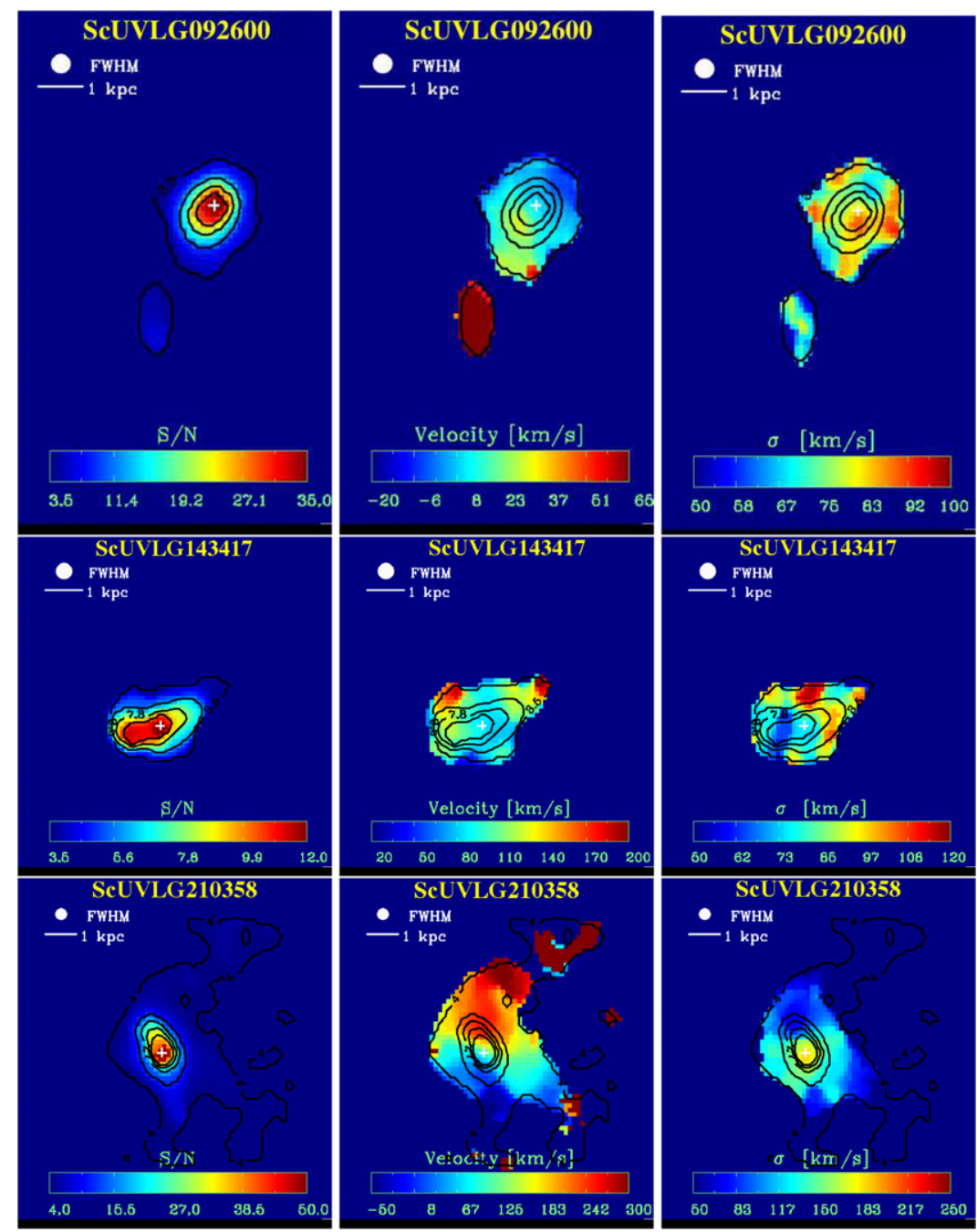

Figure 2. OSIRIS IFS results for three ScUVLGs- ScUVLG092600 (top), ScUVLG143417 (middle row), and ScUVLG210358 (bottom). We show S/N maps (left), velocity maps (middle column), velocity dispersions (right) with $\mathrm{S} / \mathrm{N}$ contours overlaid and nucleus (center of the brightest region) displayed as a white triangle. Contour levels correspond with marked values of the $\mathrm{S} / \mathrm{N}$ color bar. The smoothed PSF and physical scale are shown at the upper left.

suggesting dispersion-dominated systems rather than smoothly rotating thin disks.

\subsection{ScUVLG092600}

The bright component in the NW of this system has a weak, but regular velocity structure across its disk. HST $\mathrm{H} \alpha$ imaging reveals a filamentary component extending into the southwest, potentially suggesting an outflow. Since this feature is undetected in optical or UV morphologies, it is unlikely to be a tidal feature or spiral arm. The mild velocity gradient may arise from an outflow in the southwestern region of the disk, rather than from smooth rotation. For a rotating disk scenario, we find an inclination, $i=40 \pm 5^{\circ}$. The bottom panel of Figure 3 displays the PV diagram, sliced along the central region of the disk (shown in magenta on the velocity field, at top). We determine a circular velocity of $v_{c}=\frac{20 \mathrm{~km} \mathrm{~s}^{-1}}{\sin i} \approx 31_{-3}^{+4} \mathrm{~km} \mathrm{~s}^{-1}$. Qualitatively, the PV diagram shows that velocity dispersion dominates the velocity structure across the disk. Quantitatively, the integrated spectrum gives $\sigma=84 \pm 3 \mathrm{~km} \mathrm{~s}^{-1}$, and $v / \sigma$ for this component is $0.37 \pm 0.05$. Assuming that the observed velocity dispersion of the ionized gas for this component can provide an estimate for the disk's dynamical mass, $M_{\mathrm{dyn}}$, we use the following relation:

$$
M_{\mathrm{dyn}}=\frac{C \sigma^{2} R}{G},
$$

where $C$ is the geometric constant ( $C=3.4$ for thin rotating disks, $C=5$ for spherical geometry), $G$ is the gravitational constant, $R$ is the radius of the disk, and $\sigma$ is the velocity dispersion. Adopting the following values: $R=0.3=0.9$ $\mathrm{kpc}, \sigma=84 \mathrm{~km} \mathrm{~s}^{-1}$, we determine $M_{\mathrm{dyn}}=4.4-6.5 \times 10^{9} M_{\odot}$, corresponding to $3.4<C<5$.

The companion in the southeast, spanning few velocity resolution units across, has systemic velocity of $\Delta v=$ $+80 \mathrm{~km} \mathrm{~s}^{-1}$ compared to the brighter component. While we do not detect any ionized gas linking these two components, they are likely in the process of interacting given their close proximity $(\leqslant 3 \mathrm{kpc}$ ). As further evidence of interaction, T. S. Gonçalves et al. (2009, in preparation) find that the bright component's central region is consistent with regular rotation, while outer edges become more disturbed and dispersion dominated.

\subsection{ScUVLG143417}

This galaxy's morphology differs from ScUVLG092600, with two bright cores (separated by $<1 \mathrm{kpc}$ ) embedded in a 


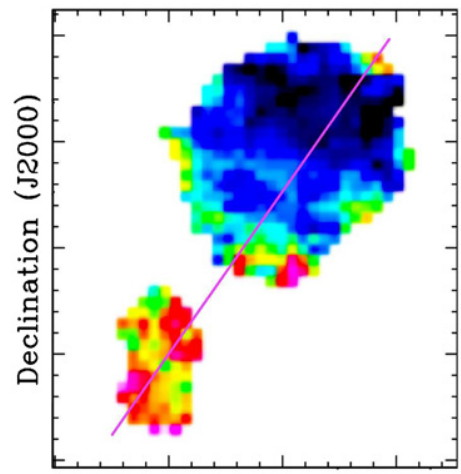

Right Ascension (J2000)

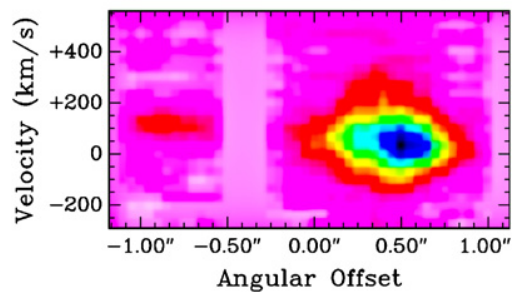

Figure 3. PV diagram for ScUVLG092600 is shown at the bottom for a slice along the velocity gradient (marked in magenta, on the velocity field, at the top). The PV diagram illustrates a slight velocity gradient with high dispersion.

fainter, disturbed (irregular and asymmetric) galaxy. We cannot detect the fainter arms very clearly in the $\mathrm{Pa} \alpha$ data. However, the central region surrounding the brightest two star-forming knots does appear kinematically disturbed-lacking structure in velocity and velocity dispersion.

While a face-on disk could explain the absence in the velocity structure, the lack of a structure in $\sigma$ makes that scenario less plausible. Therefore, inclination is unconstrained. Using the integrated spectrum, we find $\sigma=86 \pm 3 \mathrm{~km} \mathrm{~s}^{-1}$. With no observed velocity gradient, we can only place an upper limit: $v / \sigma<0.7$, using $v_{c}=\left(v_{\max }-v_{\min }\right) / 2$ and no inclination correction. This case represents disturbed kinematics with multiple cores and underlying faint features, suggestive of recent merger activity.

\subsection{ScUVLG210358}

While morphologically similar to ScUVLG143417, we find that kinematics in this galaxy are quite different. Again, the optical image reveals faint, irregular, and asymmetric arms with a bright, unresolved star-forming core. However, in this case, there is a single bright, symmetric core. This galaxy is one of the most massive and luminous ScUVLGs in our sample. The position angle for the $\mathrm{Pa} \alpha$ isophotes (from the $\mathrm{S} / \mathrm{N}$ contours) does not align with that of the velocity field, possibly indicating the presence of a warp or bar. The velocity gradient across the highest $\mathrm{S} / \mathrm{N}$ region of this galaxy is very regular, maintaining a similar structure to the outer, fainter regions of the system. Hints of a merger are apparent from HST images, displaying irregular, clumpy light distribution and a potential faint companion to the southwest. Yet the dynamics of this galaxy's central region show ordered rotation, with the $\sigma$ peak corresponding with the galaxy's nucleus. Therefore, this galaxy has dynamics consistent with disks. We find an inclination of $i=51 \pm 4^{\circ}$, circular velocity of $v_{c}=\frac{145 \mathrm{~km} \mathrm{~s}^{-1}}{\operatorname{sini}}=186_{-9}^{+12} \mathrm{~km} \mathrm{~s}^{-1}$, and $\sigma=175 \pm 4 \mathrm{~km}$ $\mathrm{s}^{-1}$, giving $v / \sigma \stackrel{\sin i}{\approx} 1.1$. Using Equation (1), at $R=1.5 \mathrm{kpc}$, we derive $M_{\text {dyn }}=4 \times 10^{10} M_{\odot}($ for $C=3.4)$ and $6 \times 10^{10} M_{\odot}$ (for $C=5$ ). Similar to ScUVLG092600, the signature for rotation is present, yet, this system is marginally dispersion dominated.

\subsection{Simulation to High-z}

To best compare our results with high- $z$ IFS observations (tracing $\mathrm{H} \alpha$ emission in the $K_{s}$-band), we perform artificial redshifting to determine how our three galaxies would appear if placed at $z \sim 2$. Using the recovered flux, velocity, and dispersion maps described in Section 3, we generate a synthetic model data cube which is rebinned to the angular scale which would be observed at redshift $z=2.2645$ (chosen so that $\mathrm{H} \alpha$ emission in $K_{s}$ band avoids strong $\mathrm{OH}$ emission features). Assuming that $\mathrm{H} \alpha$ emission traces $\mathrm{Pa} \alpha$, we normalize this model to the total $\mathrm{H} \alpha$ luminosity determined by the unresolved SDSS spectroscopic data and artificially "observe" the model galaxy using the OSIRIS simulation code of Law et al. (2006) and data reduction and analysis techniques of Law et al. (2007). We refer the reader to these papers for a full description of the routines, noting that the simulation code fully accounts for the characteristics of the OSIRIS+LGSAO system and has been shown to well represent actual IFS observations of highredshift galaxies (Law et al. 2007). Each of our three galaxies is artificially redshifted and re-observed, using a simulated 50 mas lenslet scale in $K_{S}$ band for a total of $2 \mathrm{hr}$ of integration time under typical atmospheric conditions. These parameters are chosen to represent typical observational values used in Law et al. (2007) and D. R. Law et al. (2009, in preparation).

We show the high- $z$ simulated data in Figure 4, with black contours marking the $z \sim 0.2$ data. For example, the middle panels display high- $z$ velocity with low- $z$ velocity (see data in Figure 2) contours overlaid. In all cases, the high- $z$ data match the brightest low- $z$ features. However, this figure illustrates some of the difficulties of high-redshift LBG observations: resolution loss in the simulated S/N data causes ScUVLG092600 to appear more face-on; the simulated $\sigma$-map of ScUVLG092600 appears more disturbed as edge features dominate; lower resolution blends the double core of ScUVLG143417 into a single blob; selecting velocities where $\mathrm{S} / \mathrm{N}$ exceeds detectable limits gives the velocity map in ScUVLG143417 a slightly more regular appearance; surface brightness dimming hides the complex fainter structure in ScUVLG210358. We derive values for axis ratios, inclination, circular velocity, $\sigma$ and $v / \sigma$ from the high- $z$ simulated data (shown in Table 1, alongside the observed low- $z$ results).

The $v / \sigma$ for high- $z$ simulations of ScUVLG092600, ScUVLG143417, and ScUVLG210358 are $1.0_{-0.6}^{+0.3}, 0.4 \pm 0.1$ (no inclination correction), and $0.9 \pm 0.1$, respectively. Therefore, in all the cases, $v / \sigma$ in high- $z$ simulations differs somewhat from low $-z$ values (as shown in Table 1). For ScUVLG092600, $v / \sigma$ increases in the high- $z$ simulated case, while decreasing for the other two galaxies. We find that the high- $z$ simulations physically resemble and have similar, though slightly higher, $v / \sigma$ values as $z=2$ LBGs, which have $v / \sigma \sim 0.3-0.7$ (D. R. Law et al. 2009, in preparation).

\section{DISCUSSION AND SUMMARY}

We are pioneering a new study of the dynamics and kinematics in local $(z<0.3)$ UV-selected galaxies that resemble LBGs in most physical properties. Similar studies have yielded mixed results about LBG dynamics at high- $z$, but our study of three low- $z$ ScUVLGs shows that dispersion-dominated kinematics is a viable scenario. Although two of the three cases show some signs of smooth rotation, $v / \sigma \lesssim 1$ in these galaxies. Since HST 


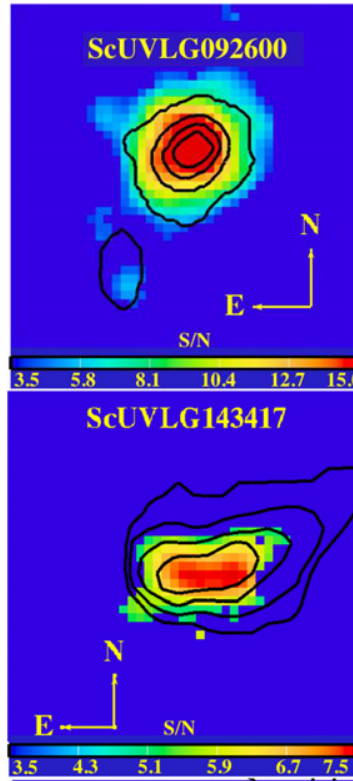

ScUVLG210358

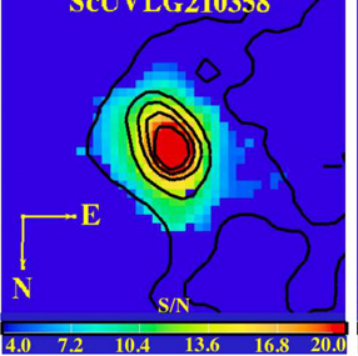

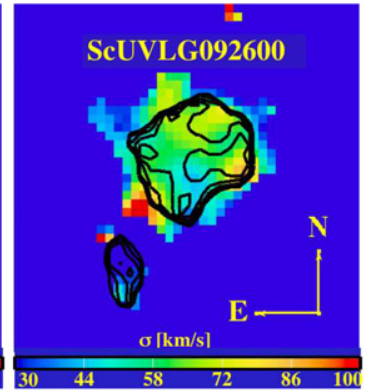

ScUVLG143417

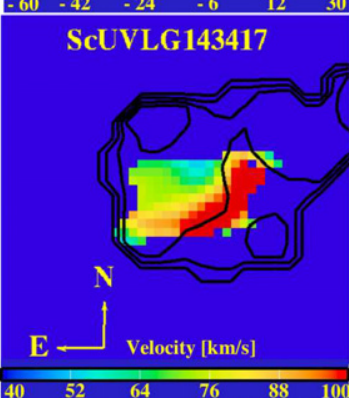

ScUVLG210358

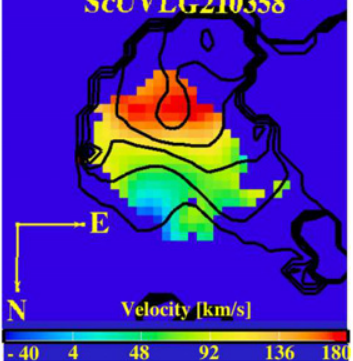

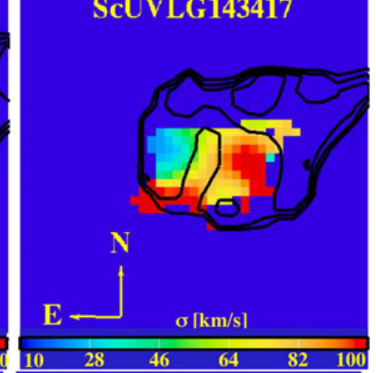

ScUVLG210358

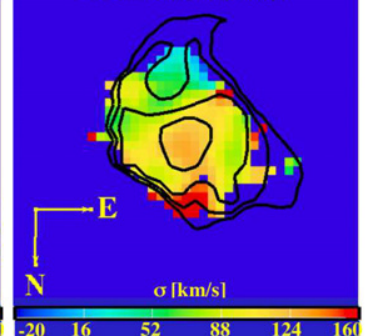

Figure 4. High- $z$ simulations for $\mathrm{S} / \mathrm{N}$, velocity, and $\sigma$ maps (from the left to right column) for ScUVLG092600 (top), ScUVLG143417 (middle row), and ScUVLG210358 (bottom). Overlying black contours outline data from Figure 2, such that contours show low- $z$ velocity data in the middle column and low- $z$ $\sigma$ data in the right column, with levels matching marked values from color bars in Figure 2.

images show disturbed morphologies, nearby companions, and evidence of ongoing interactions (tidal features, plumes, etc.), it is not surprising that velocity dispersions in these galaxies are so high.

IFS studies of dynamics in LBGs draw conclusions ranging from smooth rotation to dispersion-dominated kinematics. Genzel et al. (2008) study a sample of massive $\left(M \sim 10^{11} M_{\odot}\right)$, $z=2-3$ rest frame UV-selected galaxies and classify these as turbulent, clumpy disks with formed bulges, undergoing secular evolution. Studying a subset of LBGs having the highest velocity fields, Förster Schreiber et al. (2006) find that the majority exhibit smooth rotation, consistent with cosmological predictions of flattened disk-like galaxies (Mo et al. 1998), while a more comprehensive sample demonstrates larger contributions from both major mergers and high dispersion systems (Förster Schreiber et al. 2009). Nesvadba et al. (2006) and Stark et al. (2008) determine gravitationally lensed LBGs at $z=3.2$ and 3.07, respectively, are examples of rotationally supported disks. Wright et al. (2007) fit their $z=1.5$ LBG with a rotation model but cannot exclude a merger scenario. D. R. Law et al. (2009, in preparation) argue for largely dispersion dominated kinematics in their sample of LBGs. Analyzing IFS data for one massive LBG at $z=3.2$, Nesvadba et al. (2008) find evidence for dispersion-dominated kinematics.

Comparing our sample of LBG analogs to LBGs at high$z$, our results are more consistent with compact, kinematically disturbed systems (i.e., similar to D. R. Law et al. 2009, in preparation, and see discussion by Förster Schreiber et al. 2009) rather than larger, more massive disks (i.e., Genzel et al. 2008; Förster Schreiber et al. 2006). While our selection criteria for ScUVLGs include compactness of the UV-emitting region, HST images do reveal underlying low surface brightness, extended structure in two cases-ScUVLG1434 and ScUVLG2103. These two examples represent the most massive ScUVLGs. Meanwhile, for high- $z$ observations, optimization of candidates may bias this study by selecting larger and brighter galaxies and may not represent the general physics governing galaxy behavior at these redshifts (Nesvadba et al. 2008; Stark et al. 2008). Our view at low- $z$ may provide insight about smaller, more compact, $z=2-3$ LBGs. In these low- $z$ cases, we argue that mergers may be responsible for the observed kinematics and morphologies and speculate that a similar scenario driving LBG characteristics at high- $z$ cannot be excluded by current data (see also Overzier et al. 2008).

Lehnert et al. (2009) investigate other mechanisms capable of causing high dispersions in high- $z$ star-forming galaxies. They find that gas accretion cannot sustain the observed high dispersions. Studying the relationship between velocity dispersion and emission-line characteristics, they conclude intense star formation can account for high dispersions in these systems through a feedback cycle between turbulence in the interstellar medium (ISM) and star formation. The proposed scenario may offer a plausible explanation for observed gas kinematics once galaxies reach a turbulent phase and associated starburst (Lehnert et al. 2009). 
We show that simulated high- $z$ data produce $v / \sigma$ values somewhat different from actual values. Misinterpretation of kinematic features may result from surface brightness and resolution effects. However, these simulations do resemble $z=2$ LBGs studied by D. R. et al. (2009, in preparation) in physical appearance and $v / \sigma$.

Having launched this pilot study, we will continue researching a larger sample of ScUVLGs-performing kinemetry analysis (Shapiro et al. 2008) in order to draw statistically robust conclusions about the dynamics of these low- $z$, high SFR, UV-selected galaxies (see T. S. Gonçalves et al. 2009, in preparation). Detailed IFS studies of local LBG analogs offer a unique method for capturing important physics-pinpointing the mechanisms most generally responsible for the high starformation phase occurring in LBGs at high- $z$ and in ScUVLGs at low- $z$.

We thank the referee for helpful and insightful comments. The authors wish to recognize and acknowledge the very significant cultural role and reverence that the summit of Mauna Kea has always had within the indigenous Hawaiian community. We are most fortunate to have the opportunity to conduct observations from this mountain. A.R.B. gratefully recognizes Dr. Jacqueline van Gorkom for her advice.

\section{REFERENCES}

Abadi, M. G., Navarro, J. F., Steinmetz, M., \& Eke, V. R. 2003, ApJ, 591,499
Baldwin, J. A., Phillips, M. M., \& Terlevich, R. 1981, PASP, 93, 5

Basu-Zych, A. R., et al. 2007, ApJS, 173, 457

Best, P. N., Kauffmann, G., Heckman, T. M., \& Ivezić, Ž. 2005, MNRAS, 362,9

Bouwens, R. J., Illingworth, G. D., Blakeslee, J. P., \& Franx, M. 2006, ApJ, 653,53

Förster Schreiber, N. M., et al. 2006, ApJ, 645, 1062

Förster Schreiber, N. M., et al. 2009, in press, arXiv:astroph/0903.1872

Genzel, R., et al. 2008, ApJ, 687, 59

Gooch, R. 1996, in ASP Conf. Ser. 101, Astronomical Data Analysis Software and Systems V, ed. G. H. Jacoby \& J. Barnes (San Francisco, CA: ASP), 80

Heckman, T. M., et al. 2005, ApJ, 619, L35

Hoopes, C. G., et al. 2007, ApJS, 173, 441

Kauffmann, G., et al. 2003, MNRAS, 341, 33

Kormendy, J., \& Fisher, D. B. 2005, in Rev. Mex. Astron. Astrofis. Conf. Ser. 23 (Rev. Mex. Astron. Astrofis., Vol. 27), ed. S. Torres-Peimbert \& G. MacAlpine, 101

Kroupa, P. 2001, MNRAS, 322, 231

Larkin, J., et al. 2006, in Proc. SPIE 6269, 62691A

Law, D. R., et al. 2007, ApJ, 669, 929

Law, D. R., Steidel, C. C., \& Erb, D. K. 2006, AJ, 131, 70

Lehnert, M. D., et al. 2009, in press, arXiv:astroph/0902.2784

Mo, H. J., Mao, S., \& White, S. D. M. 1998, MNRAS, 295, 319

Nesvadba, N. P. H., et al. 2006, ApJ, 650, 661

Nesvadba, N. P. H., Lehnert, M. D., Davies, R. I., Verma, A., \& Eisenhauer, F. 2008, A\&A, 479, 67

Overzier, R. A., et al. 2008, ApJ, 677, 37

Salim, S., et al. 2005, ApJ, 619, L39

Shapiro, K. L., et al. 2008, ApJ, 682, 231

Stark, D. P., Swinbank, M., Ellis, R. S., Dye, S., Smail, I. R., \& Richard, J. 2008, Nature, 455, 775

Steidel, C. C., et al. 2000, ApJ, 532, 170

van Dam, M. A., et al. 2006, PASP, 118, 310

White, S. D. M., \& Rees, M. J. 1978, MNRAS, 183, 341

Wizinowich, P. L., et al. 2006, PASP, 118, 297

Wright, S. A., et al. 2007, ApJ, 658, 78 